\title{
Effect of 3 T MRI on the function of shunt valves-Evaluation of Paedi GAV, Dual Switch and proGAV
}

\author{
D. Lindner ${ }^{\mathrm{a}, *}$, C. Preul ${ }^{\mathrm{b}}$, C. Trantakis ${ }^{\mathrm{a}}$, H. Moeller ${ }^{\mathrm{b}}$, J. Meixensberger $^{\mathrm{a}}$ \\ ${ }^{a}$ Department of Neurosurgery, University of Leipzig, Liebigstraße 20, 04103 Leipzig, Germany \\ ${ }^{\mathrm{b}}$ Max-Planck-Institut for Human Cognitive and Brain Sciences, Stephanstr. 1a, 04103 Leipzig, Germany
}

Received 3 January 2005; received in revised form 14 March 2005; accepted 16 March 2005

\begin{abstract}
The MR-compatibility of medical implants and devices becomes more and more important with the increasing number of high-field MRscanners employed. Until the end of 2004, about twenty 3 T MR in Germany will be in clinical practice. Patients with hydrocephalus need frequent follow-up MR-examinations to assure correct functioning of a shunt. We tested three types of gravitational valves: the Paedi GAV, the Dual Switch and as a new programmable valve the proGAV (Miethke Company, Berlin), that have not been evaluated at $3 \mathrm{~T}$, yet. In sum, there is strong evidence for maintenance of function of these valves after exposure to $3 \mathrm{~T}$. This also implies the programmable valve, as long as the brake mechanism is properly adjusted during MR-examination.
\end{abstract}

(c) 2005 Elsevier Ireland Ltd. All rights reserved.

Keywords: MRI; Paedi GAV; Dual Switch and proGAV

\section{Introduction}

There is no universal protocol for the assessment of MRcompatibility of shunt valves in terms of function and the apparatus to characterize the hydrodynamic performance of valves after exposure to magnetic fields differs among manufacturers [1,2]. In Leipzig, we overview in the group of patients who were treated by programmable Codman Medos-P valve one patient with not adjustable device after applying $3 \mathrm{~T}$ magnetic field. So, we had to exchange the valve. That is why we excluded programmable valves from application in $3 \mathrm{~T}$ MR and initiated, together with Miethke-Aesculap, our study to evaluate independently their typical gravitational valves in this high magnetism field. In 2003, the Dual Switch and Paedi GAV were evaluated in the Shunt Evaluation Laboratory in Cambridge [4]. We employed their protocol for this study as well and added a programmable variant of a shunt (the proGAV) as data on changes in function are not available yet. We addressed the functioning of the valve in

\footnotetext{
* Corresponding author. Tel.: +49 341971500; fax: +49 3419717509.

E-mail address: dlind@ medizin.uni-leipzig.de (D. Lindner).
}

particular, as we believe that heating effects or displacement effects are negligible. On the one hand, because the material of the shunts does not have ferromagnetic properties, on the other hand because the valve is in vivo well fixed underneath the scalp $[6,8,9,18]$.

\section{Material and methods}

The hydrodynamic performance of three Dual Switch valves, three Paedi GAV valves and three proGAV valves was evaluated before and after exposure to $3 \mathrm{~T}$.

Valve characteristics:

1. Dual Switch: The Dual Switch valve is a combination of a ball-in-cone valve and a membrane valve. It is designed for adult patients. Two small titanium plates in the middle of the diaphragms together with the titanium sphere fixed in the housing act [19]. The opening pressure of different valve-chambers is determined by two different springs for horizontal and vertical position. All materials (titanium, tantalum, silicone) are non-ferromagnetic [4,11-13]. 
2. Paedi GAV: The Paedi GAV is a gravitational valve dedicated for management of paediatric hydrocephalus. The inlet valve is a ball-on-spring mechanism, the outlet is backed by a tantalum ball, which increases the operational pressure in upright body position. It is available in six performance pressure ranges $[4,15]$.

3. proGAV: The proGAV is a programmable valve designed for adults and children. It contains an adjustable safety brake mechanism. After releasing the brake, the opening pressure (in a range from 0 to $20 \mathrm{~cm} \mathrm{H}_{2} \mathrm{O}$ ) can be adjusted; a rotor with two magnets inside the valve can be switched from outside by a magnetic stream. All other materials (titanium, sapphire) are completely non-ferromagnetic. One of the proGAV was tested without brake (No. SN 01004 8) to serve as a negative control.

\section{Assessment of valve function}

\subsection{Test protocol}

First, we developed a standard protocol to investigate the valves before and after the MRI scan. Measure equipment and test procedures were provided by C. Miethke GmbH \& Co. Evaluations were analysed in a typical producing protocol comparable the standard ASTM F647.

\subsubsection{Test equipment}

A roll-pump delivers a constant flow of water or air through the valve in an adjustable range of $5-50 \mathrm{ml} / \mathrm{h}$.

The valve is attached to a test dummy on a tip-over table. A computerized system measured the opening pressure permanently with a tolerance of $\pm 0.5 \mathrm{~cm} \mathrm{H}_{2} \mathrm{O}$ (standard deviation) (Fig. 1).

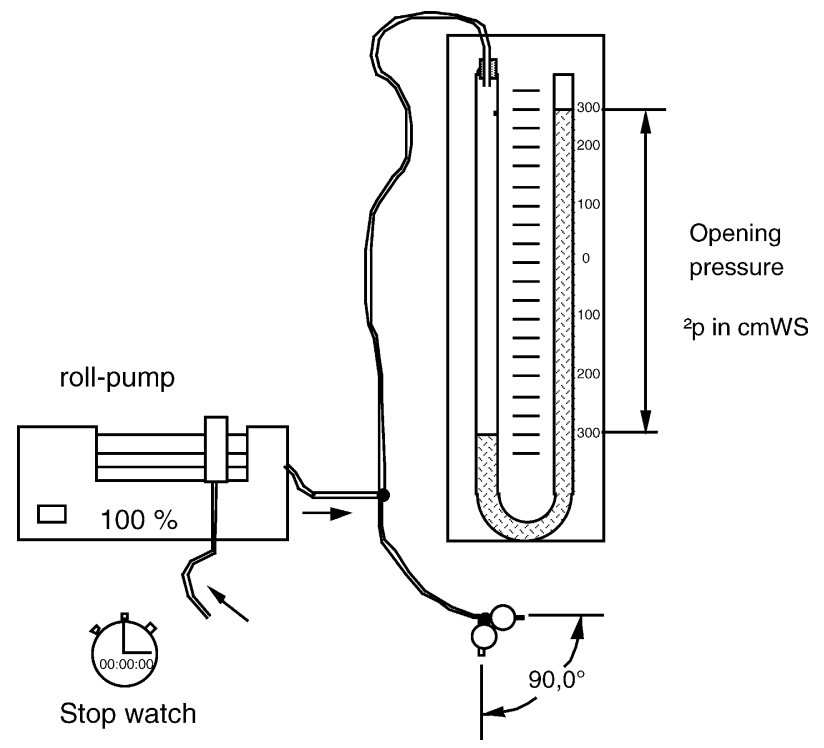

Fig. 1. Air flow measure tool.
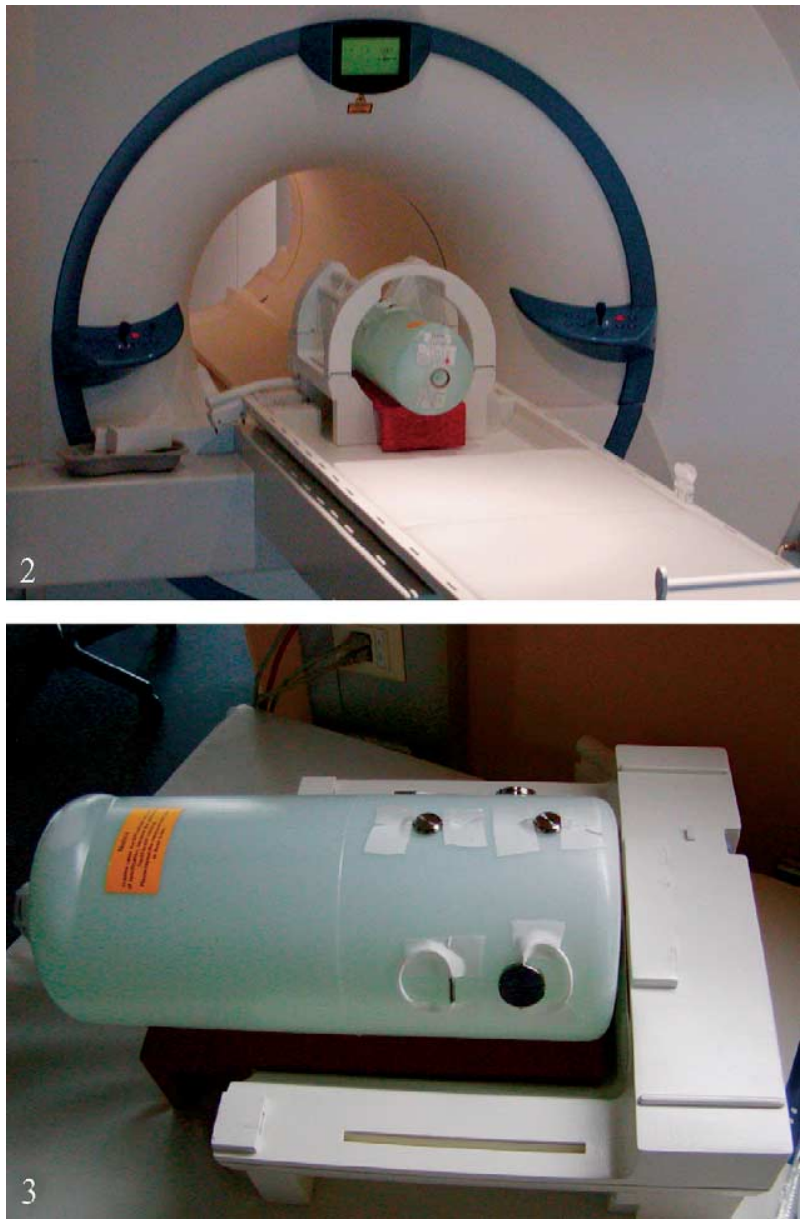

Figs. 2 and 3. Adjustment of the valves on the phantom model in the $3 \mathrm{~T}$ MR (Siemens).

In addition, the air flow records the density of the air at valve closure and the water flow test measures the pressure of the ventricle [17].

\subsection{MR-scanning protocol}

After completion of the pre-scan tests, the valves underwent MRI-scan. All magnetic resonance imaging (MRI) experiments were performed on a 3-T human-scale whole-body scanner (MAGNETOM Trio, Siemens, Erlangen, Germany) using a circularly polarized head coil of birdcage design (Bruker BioSpin, Ettlingen, Germany) and a cylindrical water phantom provided by the manufacturer (length: $37 \mathrm{~cm}$; diameter: $17 \mathrm{~cm}$; mixture of $1.24 \mathrm{~g} \mathrm{NiSO}_{4} \cdot 6 \mathrm{H}_{2} \mathrm{O}$ and $2.62 \mathrm{~g}$ $\mathrm{NaCl}$ per liter of pure water). All valves were mounted on the side of the phantom (Figs. 2 and 3).

For MRI scanning experiments, two valves of every kind were aligned parallel to the axis of the main magnetic field, $\mathrm{B}_{0}$ (i.e., along the $z$-axis) and one of every perpendicularly to $\mathrm{B}_{0}$.

The MRI protocol is analogous to clinical practice and consists of a scout, $T_{2}$-weighted (T2w) two-dimensional 
(2D) spin-echo (SE) imaging, $T_{1}$-weighted $(\mathrm{T} 1 \mathrm{w})$ threedimensional (3D) gradient-recalled echo (GRE) imaging and diffusion-weighted (dw) 2D echo planar imaging (EPI). For T2w-imaging, rapid acquisition with relaxation enhancement (RARE) [7] sequences (echo-train length: 13; repetition time, $T_{\mathrm{R}}: 6.2 \mathrm{~s}$; echo time, $T_{\mathrm{E}}: 108 \mathrm{~ms}$; refocusing pulse flip angle, $\square: 150^{\circ}$; acquisition bandwidth: $107 \mathrm{~Hz} /$ pixel; 25 slices, thickness: $2 \mathrm{~mm}$, inter-slice distance: $6 \mathrm{~mm} ; 2$ acquisitions) were acquired with sagittal (field of view, FOV: $220 \mathrm{~mm} \times 220 \mathrm{~mm}$; matrix: $512 \times 410$; phase-encoding along the anterior-posterior direction), coronal (FOV: $220 \mathrm{~mm} \times 175 \mathrm{~mm}$; matrix: $512 \times 326$; phaseencoding along the right-left direction) and axial slice orientation (identical parameters as for the coronal scan). A sagittal 3D slab (thickness: $176 \mathrm{~mm}$ ) was acquired for T1wimaging with magnetization-prepared rapid gradient echo (MP-RAGE) [14] with selective water excitation and linear phase encoding $\left(T_{\mathrm{R}}: 1.3 \mathrm{~s}\right.$ for the total sequence cycle; $T_{\mathrm{R}}{ }^{\prime}: 10 \mathrm{~ms}$ for the GRE kernel; $T_{\mathrm{E}}: 3.93 \mathrm{~ms}$; excitation pulse flip angle, $\square: 10^{\circ}$; acquisition bandwidth: $130 \mathrm{~Hz} /$ pixel; FOV: $256 \mathrm{~mm} \times 240 \mathrm{~mm}$; matrix: $256 \times 240$; 176 partitions; $70 \%$ slice resolution; 2 acquisitions). Magnetization preparation consisted of a non-selective inversion pulse (inversion time, TI: $650 \mathrm{~ms}$ ). For dw-imaging, a SE-EPI sequence [10] with fat suppression was used to acquire 44 axial slices (thickness: $3 \mathrm{~mm}$; inter-slice distance: $3.3 \mathrm{~mm}$; TR: $8.1 \mathrm{~s}$; TE: $120 \mathrm{~ms}$; acquisition bandwidth: $1396 \mathrm{~Hz} /$ pixel; FOV: $220 \mathrm{~mm} \times 220 \mathrm{~mm}$; matrix: $128 \times 80$; echo position at $37.5 \%$; 3 acquisitions). Diffusion weighting along 12 directions was achieved by a pair of trapezoidal gradient lobes sandwiching the $180^{\circ}$ refocusing pulse to yield $b$-factors of 0 and $1000 \mathrm{~s} / \mathrm{mm}^{2}$. The valves remained in the magnet for $45 \mathrm{~min}$ with a total scanning time of $34: 57 \mathrm{~min}$. To study the effect of the static main magnetic field separately, another set of three valves was mounted on the phantom along the $z$-axis in a second experiment and remained in the magnet for 15 min without application of radio-frequency or gradient pulses.

Afterwards the valves were sent back for re-test of functioning. The test-lab staff was blinded to which of the scanning protocols was applied to a particular valve.

\section{Results}

The results from pre-scan and post-scan measurements were calculated as a mean value from three single measurements. The series of measurements are available for every valve and demonstrate individual differences in tolerable ranges, depending on the valve type and the influence of flow and reflux. Tables 1 and 2 depict the differences between the two measure times. Values were calculated as follows:

value $($ before MR) - value $($ after MR) $=$ difference value

The average deviation of the valves without the negative control (No. SN 010048) was $0.6 \mathrm{H}_{2} \mathrm{O} \pm 0.2$ for the air flow test and $0.7 \mathrm{H}_{2} \mathrm{O} \pm 0.2$ for the water flow test. Note, that the results for the negative control differ significantly indicating a shift of the pressure adjustment.

Table 1

Air flow test

\begin{tabular}{llcccc}
\hline Valve & No. & $\Delta$ Horizontal P5 ml/h & $\Delta$ Horizontal P50 ml/h & $\Delta$ Vertical P5 ml/h & $\Delta$ Vertical P50 ml/h \\
\hline Dual Switch & 62720202 & 0.8 & -0.5 & 0.9 & 0.2 \\
Dual Switch & 28150100 & 0.9 & 0.8 & -0.1 & 0.1 \\
Dual Switch & 14961197 & 0.4 & -0.1 & -0.5 & 0.6 \\
Paedi GAV & 304508013 & -0.5 & -0.5 & 0.2 & 0.5 \\
Paedi GAV & 394208013 & 0 & 0 & 0.5 & 0.7 \\
Paedi GAV & 222001003 & 0.2 & 0.7 & 1 & 0.5 \\
ProGAV+ & SN 010108 & 0.9 & -0.5 & -0.6 & 0.8 \\
ProGAV+ & SN 010018 & -0.8 & $\mathbf{- 1 8}$ & $-\mathbf{1 8 . 8}$ & -0.4 \\
ProGAV- & SN 010048 & $-\mathbf{1 9 . 4}$ & & & $-\mathbf{1 9 . 5}$ \\
\hline
\end{tabular}

Table 2

Water flow test

\begin{tabular}{|c|c|c|c|c|c|}
\hline Valve & No. & $\Delta$ Horizontal P5 ml/h & $\Delta$ Horizontal $\mathrm{P} 50 \mathrm{ml} / \mathrm{h}$ & $\Delta$ Vertical P5 ml/h & $\Delta$ Vertical P50 $\mathrm{ml} / \mathrm{h}$ \\
\hline Dual Switch & 62720202 & 1 & -0.5 & 0.5 & 1 \\
\hline Dual Switch & 28150100 & 1 & 1 & 1 & -1 \\
\hline Dual Switch & 14961197 & 1 & 0 & 1 & -1 \\
\hline Paedi GAV & 304508013 & -0.8 & 0 & 1 & 0.5 \\
\hline Paedi GAV & 394208013 & 1 & 0 & 0.5 & 0 \\
\hline Paedi GAV & 222001003 & 0.5 & 0.5 & 0 & 1 \\
\hline ProGAV+ & SN 010108 & -1 & -1 & -1 & -1 \\
\hline ProGAV+ & SN 010018 & -0.5 & 1 & -1 & 0 \\
\hline ProGAV- & SN 010048 & -19.5 & -18.5 & -18.5 & -18 \\
\hline
\end{tabular}




\section{Discussion}

The aim of the study was to evaluate the function of different valves after exposure to a $3 \mathrm{~T}$ MR field. The numbers of $3 \mathrm{~T}$ magnetoms in Germany will increase in the next years, necessitating more knowledge about the MR-compatibility of common devices and implants at higher field strength. We focused on the function of the valves only. Displacement $[3,5,15]$ or extensive heating of the devices could not be expected due to the chemical constituents of the shunts. The functioning of the valves is the crucial parameter. In our group of patients with hydrocephalus who received a shunt system we had one patient with problems after $3 \mathrm{~T}$. The Medos-P valve moved from 150 to 30 and was not longer adjustable after magnetic application. The valve was removed as a evidence for malfunction of a programmable valve that was known to be safe at $1.5 \mathrm{~T}$.

Most of the currently used valves may not be affected by high magnetic field strength [16]. Special care has to be taken in programmable valves. Although referring to $1.5 \mathrm{~T}$ only, Zemack and Romner described adjustment problems in $2 \%$ in a retrospective study of 583 patients who were treated with Codman Hakim programmable valve [20].

In sum, our $3 \mathrm{~T}$ magnetic field had no influence on stability and safety of the tested Miethke valves. The proGAV as a programmable valve can only be exposed to $3 \mathrm{~T}$ if the brake mechanism is safely adjusted. Otherwise, a malfunction can result that may cause severe consequences for the patient $[3,19]$.

This study adds to the knowledge about the MRcompatibility of medical implants and devices. For medicolegal reasons we refuse liability for the use of the tested valves at $3 \mathrm{~T}$. But there is strong evidence that the valves maintain function after MR scanning, given the break mechanism is properly adjusted in the proGAV.

\section{References}

[1] Aschoff A, Kremer P, Benesch C, Fruh K, Klank A, Kunze S. Overdrainage and shunt technology. A critical comparison of programmable, hydrostatic and variable-resistance valves and flowreducing devices. Childs Nerv Syst 1995;11:193-202.

[2] Aschoff A, Kremer P, Hashemi B, Kunze S. The scientific history of hydrocephalus and its treatment. Neurosurg Rev 1999;22:67-93.

[3] Boon AJ, Tans JT, Delwel EJ, et al. Dutch Normal-Pressure Hydrocephalus Study: randomized comparison of low- and mediumpressure shunts. J Neurosurg 1998;88:490-5.
[4] Czosnyka M, Czosnyka Z, Pickard JD. Evaluation report: Aesculap-Miethke dual-switch and Paedi-GAV valves, vol. 144, no. 6. Cambridge, UK: Academic Neurosurgical Unit; 2003. p. 525-38.

[5] Hanlo PW, Cinalli G, Vandertop WP, et al. Treatment of hydrocephalus determined by the European Orbis Sigma Valve II Survey: a multicenter prospective 5-year shunt survival study in children and adults in whom a flow-regulating shunt was used. J Neurosurg 2003;99:52-7.

[6] Hebb AO, Cusimano MD. Idiopathic normal pressure hydrocephalus: a systematic review of diagnosis and outcome. Neurosurgery 2001;49:1166-84.

[7] Hennig J, Nauerth A, Friedburg H. RARE imaging: a fast imaging method for clinical MR. Magn Reson Med 1986;3:823-33.

[8] Kiefer M, Eymann R, Komenda Y, Steudel WI. A grading system for chronic hydrocephalus. Zentralbl Neurochir 2003;64: $109-15$.

[9] Kiefer M, Eymann R, Meier U. Five years experience with gravitational shunts in chronic hydrocephalus of adults. Acta Neurochir (Wien) 2002;144:755-67.

[10] Mansfield P. Multi-planar image formation using NMR spin echoes. Phys C 1977;21:847-52.

[11] Meier U, Kiefer M, Sprung C. Evaluation of the Miethke Dual Switch valve in patients with normal pressure hydrocephalus. Surg Neurol 2004;61:119-27.

[12] Meier U, Mutze S. Correlation between decreased ventricular size and positive clinical outcome following shunt placement in patients with normal-pressure hydrocephalus. J Neurosurg 2004;100:1036-40.

[13] Miethke C, Affeld K. A new valve for the treatment of hydrocephalus. Biomed Tech (Berl) 1994;39:181-7.

[14] Mugler III JP, Brookeman JR. Three-dimensional magnetizationprepared rapid gradient-echo imaging (3D MP RAGE). Magn Reson Med 1990;15:152-7.

[15] Oikonomou J, Aschoff A, Hashemi B, Kunze S. New valves-new dangers? 22 valves ( 38 probes) designed in the nineties in ultralongterm tests (365 days). Eur J Pediatr Surg 1999;9(Suppl. 1): 23-6.

[16] Shellock FG. Biomedical implants and devices: assessment of magnetic field interactions with a 3 T MR system. J Magn Reson Imaging 2002;16(6):721-32.

[17] Taylor R, Czosnyka Z, Czosnyka M, Pickard JD. A laboratory model of testing shunt performance after implantation. Br J Neurosurg 2002;16:30-5.

[18] Trost HA, Sprung C, Lanksch W, Stolke D, Miethke C. Dual-switch valve: clinical performance of a new hydrocephalus valve. Acta Neurochir Suppl (Wien) 1998;71:360-3.

[19] Zeilinger FS, Reyer T, Meier U, Kintzel D. Clinical experiences with the dual-switch valve in patients with normal pressure hydrocephalus. Acta Neurochir Suppl 2000;76:559-62.

[20] Zemack G, Romner B. Seven years of clinical experience with the programmable Codman Hakim valve: a retrospective study of 583 patients. J Neurosurg 2000;92:941-8. 